\title{
RELACIÓN ENTRE TRASTORNO DISFÓRICO PREMENSTRUAL Y ANSIEDAD, DEPRESIÓN Y ESTRÉS EN ESTUDIANTES UNIVERSITARIAS DE LA UNMSM
}

\section{RELATION BETWEEN DISORDER PREMENSTRUAL DISFORIC AND ANXIETY, DEPRESSION AND STRESS IN UNIVERSITY STUDENTS OF THE UNMSM}

\author{
Marcelino Riveros Q. ${ }^{1}$, Héctor Hernández V. ${ }^{1}$, Gerardo Rivas C., Ronald Castillo N. \\ Universidad Nacional Mayor de San Marcos, Lima, Perú \\ (RECIBIDO EL 9/02/2009, ACEPTADO el 17/06/2009)
}

\begin{abstract}
RESUMEN
En la presente investigación se utilizó la escala Evaluación de Estrés (EVES); construida y validada por Laura B. Oros de Sapia y Neifert, Ivana; es una escala tipo Likerts y consta de 22 ítemes que fue aplicada a una muestra de 352 sujetos de ambos sexos de la provincia de Entre Ríos. Obteniendo un alfa de Cronbach de 0.86 para la escala total. Conformada por los siguientes factores:

F1: Déficit cognitivo y estado anímico $: 1,4,5,7,13,15,18 \quad$ Total 7 ítemes.

F2: Nerviosismo e incapacidad para relajarse

: 3, 8, 10, 11, 17, 20, 22 Total 7 ítemes.

F3: Síntomas físicos

: 2, 6, 9, 12, 14, 16, 19, 21 Total 8 ítemes.
\end{abstract}

Sin embargo, nuestros hallazgos nos indican que los ítemes 2,6, 8, 11, 12, 17, 19, 20 no establecen diferencias significativas en las respuestas de los evaluados quedando, por lo tanto, fuera del análisis de los resultados. Por ello la escala final (EVES) quedó constituida por 14 ítemes para el estudio en nuestra población. Obteniendo alfa de Cronbach 0.82, indicando una alta confiabilidad.

A su vez estos hallazgos arrojaron los siguientes factores:

F1: Síntomas ansioso-depresivos : 3, 4, 7, 10,14, 15, 22: Total 7 ítemes.

F2: Déficit cognitivo y estado anímico : $1,5,13,18$ : Total 4 ítemes.

F3: Síntomas físicos : 9, 16, 21: Total 3 ítemes.

Como se observa en lo mencionado anteriormente, dos factores conservan su nombre inicial; habiendo variación en uno de ellos al cual se ha denominado "Síntomas ansiosodepresivos".

1 Docentes investigadores del Instituto de Investigaciones Psicológicas de la Facultad de Psicología, Universidad Nacional Mayor de San Marcos, Lima-Perú. E-mail: mriverosquiroz@yahoo.com 
Para la Escala Trastorno Disfórico Premenstrual (ETDPM), elaborada por los investigadores, cuyos ítemes fueron extraídos del DSM-IV, se obtuvo un Alfa de Cronbach igual a 0.877; considerándose un indicador altamente confiable.

Ambas escalas fueron aplicadas a un número de 230 estudiantes universitarias de la UNMSM.

Las variables consideradas en la investigación fueron: Edad cronológica, edad de menarquía, período regular, duración de período menstrual, dolores menstruales, quistes ováricos.

Las escalas ETPDM y EVES presentan una correlación de 0.653 siendo significativa. Indicándonos que a mayor puntuación en ambas escalas, existe elevado grado de desajuste emocional.

Palabras clave: Trastorno disfórico premenstrual, ansioso-depresivo, estado anímico.

\begin{abstract}
In this investigation we used the rating scale Stress (EVES), built and validated by Laura B. Sapia of gold and Neifert, Ivana; Likerts is a scale consisting of 22 items. Be applied to a sample of 352 subjects of both sexes in the province of Entre Rios. Getting a Cronbach alpha of 0.86 for the full scale. Comprised of the following factors:

F1: Cognitive deficits and mood: 1, 4, 5, 7, 13, 15, 18 Total 7 items.

F2: Nervousness and inability to relax: 3, 8, 10, 11, 17, 20, 22 Total 7 items.

F3: Physical Symptoms: 2, 6, 9, 12, 14, 16, 19, 21 Total 8 items.
\end{abstract}

However, our findings suggest that items $2.6,8,11,12,17,19,20$ no significant difference in the responses of those evaluated and there is consequently beyond the analysis of results. Thus the final scale (EVES) is made up of 14 items for study in our population. Getting Cronbach alpha 0.82 , indicating a high reliability.

In turn, these findings showed the following factors:

F1: anxious-depressive symptoms: 3, 4, 7, 10.14, 15, 22: Total 7 items.

F2: Cognitive deficits and mood: 1, 5, 13, 18: Total 4 items.

F3: Physical Symptoms: 9, 16, 21: Total 3 items.

As noted in the above two factors retained their original name, having changed into one which we called "Anxious-depressive symptoms."

Premenstrual dysphoric disorder scale (ETDPM), developed by the researchers, whose items were taken from the DSM-IV.

We obtained a Cronbach alpha equal to 0877 , considered a highly reliable indicator.

Both scales were applied to a number of 230 college students from the UNMSM.

The variables considered in the investigation were: chronological age, age at menarche, regular period, duration of menstrual period, menstrual cramps, ovarian cysts.

EVES and ETPDM scales show a correlation of 0.653 to be significant. Indicating that the higher scores on both scales, there is high degree of emotional maladjustment.

Keywords: Premenstrual dysphoric disorder, anxious-depressive mood. 


\section{INTRODUCCIÓN}

Según Lazarus (2000), el estrés es un factor muy importante para la salud psicológica, fisiológica y social. Esta afirmación explica el surgimiento de varias teorías e investigaciones acerca de este fenómeno. Al revisar la literatura se aprecia que los modelos más relevantes son tres:
A. Los que conciben al estrés como una respuesta.
B. Los que lo conciben como un estímulo.
C. Los que lo conciben como una interacción entre el individuo y el entorno.

Más allá del modelo considerado, se reconoce un consenso en afirmar que no todo tipo de estrés es perjudicial para la salud. Todo individuo para responder a las exigencias de la vida cotidiana requiere de una determinada cantidad de estrés. Este incremento es saludable, positivo y facilita la búsqueda de bienestar y realización personal. A este estrés se le denomina EUTRÉS y fue definido por Selye como:

\footnotetext{
"Situación en la que la buena forma física y el bienestar mental facilitan que el cuerpo en su conjunto adquiera y desarrollo su máximo potencial. El estado de EUTRÉS viene asociado con una gran claridad mental y unas facultades físicas máximas".
}

Existe otro tipo de estrés muy habitual que, lejos de ser saludable, resulta nocivo para la salud y provoca una sensación incomoda de malestar. En este caso estamos hablando de DISTRÉS, un tipo de estrés destructivo que puede generar angustia, dolor o ambos, Neidhardt (1989) afirma que el DISTRÉS afecta negativamente tanto al organismo como a la mente, ejerciendo una disminución en los recursos que dispone todo individuo para su afrontamiento.

La vulnerabilidad al estrés está determinada en gran medida por factores tales como, la herencia, antecedentes de enfermedades anteriores, salud física y mental, alimentación, contexto profesional y social, así como también los rasgos de personalidad. Estos factores influyen considerablemente en la sintomatología asociada al estrés y su momento de aparición.

Los síntomas físicos, emocionales y psíquicos de distrés son muy variados, estos síntomas incluyen ansiedad, depresión, hostilidad, miedo, tensión, problemas gastrointestinales como diarreas diarias o estreñimiento, falta de apetito o glotonería, fatiga intensa, insomnio, vértigo, taquicardia, boca seca, excesiva sudoración en la palma de las manos, perjuicio del funcionamiento intelectual y disminución del aprendizaje.

Es evidente la necesidad de realizar diagnósticos fiables y diagnósticos efectivos para lograr reducir y, en el mejor de los casos, eliminar la sensación angustiosa y desagradable del estrés.

Los síntomas psicológicos de trastorno disfórico premenstrual constituyen una causa frecuente de consulta en psiquiatría, enfocándose la terapéutica como si se tratara de "enfermedad depresiva", "neurosis" o "histeria". De esta manera el tratamiento se limitará a un enfoque psico-farmacológico sintomático con medicamentos antidepresivos o ansiolíticos durante meses o años. Si bien pueden aliviar parcial y temporalmente el 
TDPM, se pierde un tiempo valioso para llegar oportunamente a un tratamiento de la enfermedad ginecológica de base, que suele agravarse con el tiempo.

Se suele escuchar a la mujeres: "Me siento deprimida, nerviosa e irritable, lloro me enojo sin motivo y en la medida que se aproxima mi regla me pongo más agresiva e insoportable, además tengo dolor de cabeza, edema, distensión abdominal, molestias mamarias, frigidez, y diversos otros síntomas que me perturban"; esta sería la sintomatología más frecuente que presentan las mujeres que sufren del llamado TDPM. Este trastorno viene a ser un problema de salud pública que afecta significativamente a no menos de 25 millones de mujeres, siendo grave e invalidantes en más de 5 millones de ellas, según estudios realizados en EE. UU. Por tal motivo, la Journal of the American Medical Asociation (JAMA), órgano oficial de la sociedad médica americana, advierte sobre la urgente necesidad de efectuar mayores investigaciones en busca de un tratamiento eficaz para dicho trastorno.

Desde el punto de vista de la psicología clínica y de la salud, nosotros consideramos que si bien el TDPM tiene síntomas físicos, estos están unidos a factores psicológicos como, por ejemplo, creencias, aspecto cultural, estrés y rasgos de personalidad.

El presente estudio fue realizado en dos fases:

a) Adaptación de la escala original a nuestro contexto (ambas escalas).

b) Validación.

obteniendo lo siguiente:

- Para la Escala Trastorno Disfórico Premenstrual (ETDPM), elaborada por los investigadores, cuyos ítemes fueron extraídos del DSM-IV, se obtuvo un alfa de Cronbach igual a 0.877 ; considerándose un indicador altamente confiable.

- Para la escala EVES, se obtiene un alfa de Cronbach 0.824.

Ambas escalas fueron aplicadas a un número de 230 estudiantes universitarias de la UNMSM.

Las variables consideradas en la investigación fueron: Edad cronológica, edad de menarquia, período regular, duración de período menstrual, dolores menstruales, quistes ováricos.

Las escalas ETPDM y EVES presentan una correlación de 0.653 siendo significativa. Indicándonos que, a mayor puntuación en ambas escalas, existe elevado grado de desajuste emocional.

\section{Diseño}

El diseño de la presente investigación es correlacional.

\section{MÉTODO Y RESULTADOS}

El método utilizado en la investigación es el paquete estadístico SPSS 12.

En la Tabla N. ${ }^{o} 1$ observamos la distribución de la edad cronológica, la edad de la primera menstruación y la duración del periodo menstrual en un total de 230 estudiantes. 
Tabla N. ${ }^{0} 1$

\begin{tabular}{lccr}
\hline & $\begin{array}{c}\text { Edad } \\
\text { cronológica }\end{array}$ & $\begin{array}{c}\text { Edad primera } \\
\text { menstruación }\end{array}$ & $\begin{array}{c}\text { Duración del } \\
\text { Periodo }\end{array}$ \\
\hline Total & $\mathbf{2 3 0}$ & $\mathbf{2 3 0}$ & $\mathbf{2 3 0}$ \\
Media & 21.79 & 12.21 & 2.37 \\
Error típ. de la media & 0.210 & 0.097 & 0.036 \\
Mediana & 21.00 & 12.00 & 2.00 \\
Moda & 21 & 12 & 2 \\
Desv. típ. & 3.190 & 1.472 & 0.543 \\
Varianza & 10.175 & 2.166 & 0.295 \\
Mínimo & 16 & 9 & 1 \\
Máximo & 41 & 18 & 3 \\
\hline
\end{tabular}

En la Tabla N. ${ }^{\circ} 2$ se aprecia que las mujeres evaluadas presentan un mayor porcentaje en ciclo regular desde los 19-23 años de edad. Se debe informar las acciones psicológicas para que dicho ciclo no se vea alterado con manifestaciones de un desajuste emocional; de esta manera estaremos realizando prevención primaria.

Tabla N. ${ }^{0} 2$

\begin{tabular}{|c|c|c|c|c|}
\hline & & \multicolumn{2}{|c|}{ Ciclo regular } & \multirow{2}{*}{ Total } \\
\hline & & Sí & No & \\
\hline & 16 & 1 & 0 & 1 \\
\hline & 17 & 0 & 1 & 1 \\
\hline & 18 & 9 & 4 & 13 \\
\hline$E$ & 19 & 22 & 7 & 29 \\
\hline $\mathrm{D}$ & 20 & 21 & 19 & 40 \\
\hline \multirow{2}{*}{$\begin{array}{l}\text { A } \\
\text { D }\end{array}$} & 21 & 33 & 17 & 50 \\
\hline & 22 & 24 & 9 & 33 \\
\hline C & 23 & 17 & 4 & 21 \\
\hline $\mathrm{R}$ & 24 & 6 & 2 & 8 \\
\hline $\mathrm{O}$ & 25 & 7 & 3 & 10 \\
\hline $\begin{array}{l}\mathrm{N} \\
\mathrm{O}\end{array}$ & 26 & 5 & 2 & 7 \\
\hline $\mathrm{L}$ & 27 & 3 & 2 & 5 \\
\hline Ó & 28 & 4 & 0 & 4 \\
\hline G & 29 & 1 & 0 & 1 \\
\hline $\begin{array}{l}1 \\
\mathrm{C}\end{array}$ & 30 & 2 & 0 & 2 \\
\hline \multirow[t]{4}{*}{ A } & 32 & 0 & 1 & 1 \\
\hline & 33 & 2 & 0 & 2 \\
\hline & 36 & 1 & 0 & 1 \\
\hline & 41 & 1 & 0 & 1 \\
\hline tal & & 159 & 71 & 230 \\
\hline
\end{tabular}


La Tabla N. ${ }^{\circ} 3$ nos indica que en la muestra hay predominio de dolores menstruales en las mujeres cuyas edades van desde 19-23 años de edad. Esto nos confirma la acción preventiva indicada anteriormente.

Tabla N. ${ }^{0} 3$

\begin{tabular}{|c|c|c|c|c|}
\hline & & \multicolumn{2}{|c|}{ Dolores menstruales } & \multirow{2}{*}{ Total } \\
\hline & & Sí & No & \\
\hline & 16 & 1 & 0 & 1 \\
\hline & 17 & 1 & 0 & 1 \\
\hline $\mathbf{E}$ & 18 & 12 & 1 & 13 \\
\hline D & 19 & 21 & 8 & 29 \\
\hline $\mathbf{A}$ & 20 & 30 & 10 & 40 \\
\hline \multirow[t]{2}{*}{ D } & 21 & 39 & 11 & 50 \\
\hline & 22 & 22 & 11 & 33 \\
\hline C & 23 & 16 & 5 & 21 \\
\hline $\mathbf{R}$ & 24 & 6 & 2 & 8 \\
\hline $\mathbf{O}$ & 25 & 8 & 2 & 10 \\
\hline $\mathbf{N}$ & 26 & 7 & 0 & 7 \\
\hline $\mathbf{O}$ & 27 & 5 & 0 & 5 \\
\hline $\mathbf{L}$ & 28 & 3 & 1 & 4 \\
\hline $\mathbf{O}$ & 29 & 1 & 0 & 1 \\
\hline G & 30 & 1 & 1 & 2 \\
\hline I & 32 & 0 & 1 & 1 \\
\hline C & 33 & 1 & 1 & 2 \\
\hline \multirow[t]{2}{*}{$\mathbf{A}$} & 36 & 1 & 0 & 1 \\
\hline & 41 & 1 & 0 & 1 \\
\hline Total & & 176 & 54 & 230 \\
\hline
\end{tabular}

La Tabla N. ${ }^{\circ} 4$ confirma los datos estadísticos a nivel internacional que la menstruación tiene su inicio a partir de los 11 años de edad cronológica.

Tabla N. ${ }^{\circ} 4$

\begin{tabular}{lrrrr}
\hline & & \multicolumn{2}{c}{ Ciclo regular } & Total \\
\cline { 2 - 3 } & & Sí & No & \\
\hline & 9 & 2 & 3 & 5 \\
& 10 & 10 & 3 & 13 \\
& 11 & 43 & 18 & 61 \\
Edad de la & 12 & 48 & 21 & 69 \\
primera & 13 & 27 & 10 & 37 \\
menstruación & 14 & 18 & 9 & 27 \\
& 15 & 9 & 6 & 15 \\
& 16 & 1 & 1 & 2 \\
Total & 18 & 1 & 0 & 1 \\
\hline
\end{tabular}


En la Tabla N. ${ }^{\circ} 5$ se observa que a partir de los 11 hasta los 14 años de edad predominan los dolores menstruales; en este rubro se hace necesario trabajar mancomunadamente con los ginecólogos, biólogos, psiquiatras, antropólogos y psicólogos para determinar la incidencia y prevalencias de los mismos.

Tabla N. ${ }^{0} 5$

\begin{tabular}{lrrrr}
\hline & & \multicolumn{2}{c}{ Dolores menstruales } & Total \\
\cline { 2 - 4 } & & \multicolumn{1}{c}{ Sí } & No & \\
\hline & 9 & 3 & 2 & 5 \\
& 10 & 9 & 4 & 13 \\
& 11 & 50 & 11 & 61 \\
Edad & 12 & 52 & 17 & 69 \\
Primera & 13 & 27 & 10 & 37 \\
Menstruación & 14 & 23 & 4 & 27 \\
& 15 & 9 & 6 & 15 \\
& 16 & 2 & 0 & 2 \\
Total & 18 & 1 & 0 & 1 \\
\hline & & 176 & 54 & 230 \\
\hline
\end{tabular}

En más del $50 \%$ de la muestra la duración de su ciclo menstrual es de 3 a 4 días (Tabla N. ${ }^{\circ}$ ), también se hace evidente que aproximadamente un $33 \%$ no presenta un ciclo menstrual regular, lo cual será motivo de posteriores investigaciones.

Tabla N. ${ }^{0} 6$

\begin{tabular}{|c|c|c|c|c|}
\hline & & \multicolumn{2}{|c|}{ Ciclo regular } & \multirow{2}{*}{ Tota } \\
\hline & & Sí & No & \\
\hline \multirow{3}{*}{$\begin{array}{l}\text { Duración } \\
\text { Periodo }\end{array}$} & 1 a 2 días & 6 & 1 & 7 \\
\hline & 3 a 4 días & 93 & 38 & 131 \\
\hline & 5 a más & 60 & 32 & 92 \\
\hline Total & & 159 & 71 & 230 \\
\hline
\end{tabular}

En la Tabla N. ${ }^{\circ} 7$ observamos que los dolores menstruales se presentan en las mujeres cuyos periodos tienen una duración de 3-5 días o más, siendo necesario trabajar con un equipo interdisciplinario para determinar las causas psicológicas, biológicas y sociales que actúan en la persistencia de dichos dolores. 
Tabla N. ${ }^{0} 7$

\begin{tabular}{lcrrr}
\hline & & \multicolumn{2}{c}{ Dolores menstruales } & \multirow{2}{*}{ Total } \\
\cline { 3 - 4 } & & Sí & \multicolumn{1}{c}{ No } & \\
\hline \multirow{3}{*}{ Duración } & 1 a 2 días & 7 & 0 & 7 \\
Periodo & 3 a 4 días & 99 & 32 & 131 \\
& 5 a más & 70 & 22 & 92 \\
\hline Total & & $\mathbf{1 7 6}$ & $\mathbf{5 4}$ & $\mathbf{2 3 0}$ \\
\hline
\end{tabular}

Análisis de confiabilidad de la prueba

Estadísticos de fiabilidad de ETDPM

\begin{tabular}{cc}
\hline Alfa de Cronbach & N. $^{\mathbf{0}}$ de elementos \\
\hline .877 & 16 \\
\hline
\end{tabular}

Alta confiabilidad.

Estadísticos total-elemento

\begin{tabular}{ccccc}
\hline & $\begin{array}{c}\text { Media de la escala } \\
\text { si se elimina el } \\
\text { elemento }\end{array}$ & $\begin{array}{c}\text { Varianza de la } \\
\text { escala si se elimina } \\
\text { el elemento }\end{array}$ & $\begin{array}{c}\text { Correlación } \\
\text { elemento-total } \\
\text { corregida }\end{array}$ & $\begin{array}{c}\text { Alfa de Cronbach } \\
\text { si se elimina el } \\
\text { elemento }\end{array}$ \\
\hline i1 & 33.50 & 64.347 & .567 & .868 \\
i2 & 33.87 & 64.381 & .551 & .868 \\
i3 & 34.01 & 64.747 & .536 & .869 \\
i4 & 33.44 & 62.641 & .569 & .867 \\
i5 & 33.36 & 62.791 & .542 & .868 \\
i6 & 33.74 & 66.523 & .357 & .876 \\
i7 & 33.75 & 65.954 & .441 & .873 \\
i8 & 33.38 & 63.328 & .578 & .867 \\
i9 & 33.10 & 62.113 & .531 & .869 \\
i10 & 32.94 & 62.983 & .561 & .868 \\
i11 & 33.86 & 63.329 & .599 & .866 \\
i12 & 33.54 & 66.083 & .316 & .879 \\
i13 & 33.42 & 63.913 & .551 & .868 \\
i14 & 33.18 & 63.757 & .470 & .872 \\
i15 & 33.74 & 63.371 & .521 & .869 \\
i16 & 33.31 & 61.795 & .648 & .864 \\
\hline
\end{tabular}


Se observa que todos los ítemes que componen la ETDPM tienen una buena correlación, por consiguiente, la escala mide lo que dice medir.

\section{Análisis factorial ETDPM}

\section{Matriz de correlaciones (a)}

Determinante $=.002$

\section{KMO y prueba de Bartlett}

\begin{tabular}{llr}
\hline Medida de adecuación muestral de Kaiser-Meyer-Olkin. & .861 \\
Prueba de esfericidad de & Chi-cuadrado aproximado & 1370.314 \\
Bartlett & Gl & 120 \\
& Sig. & .000 \\
\hline
\end{tabular}




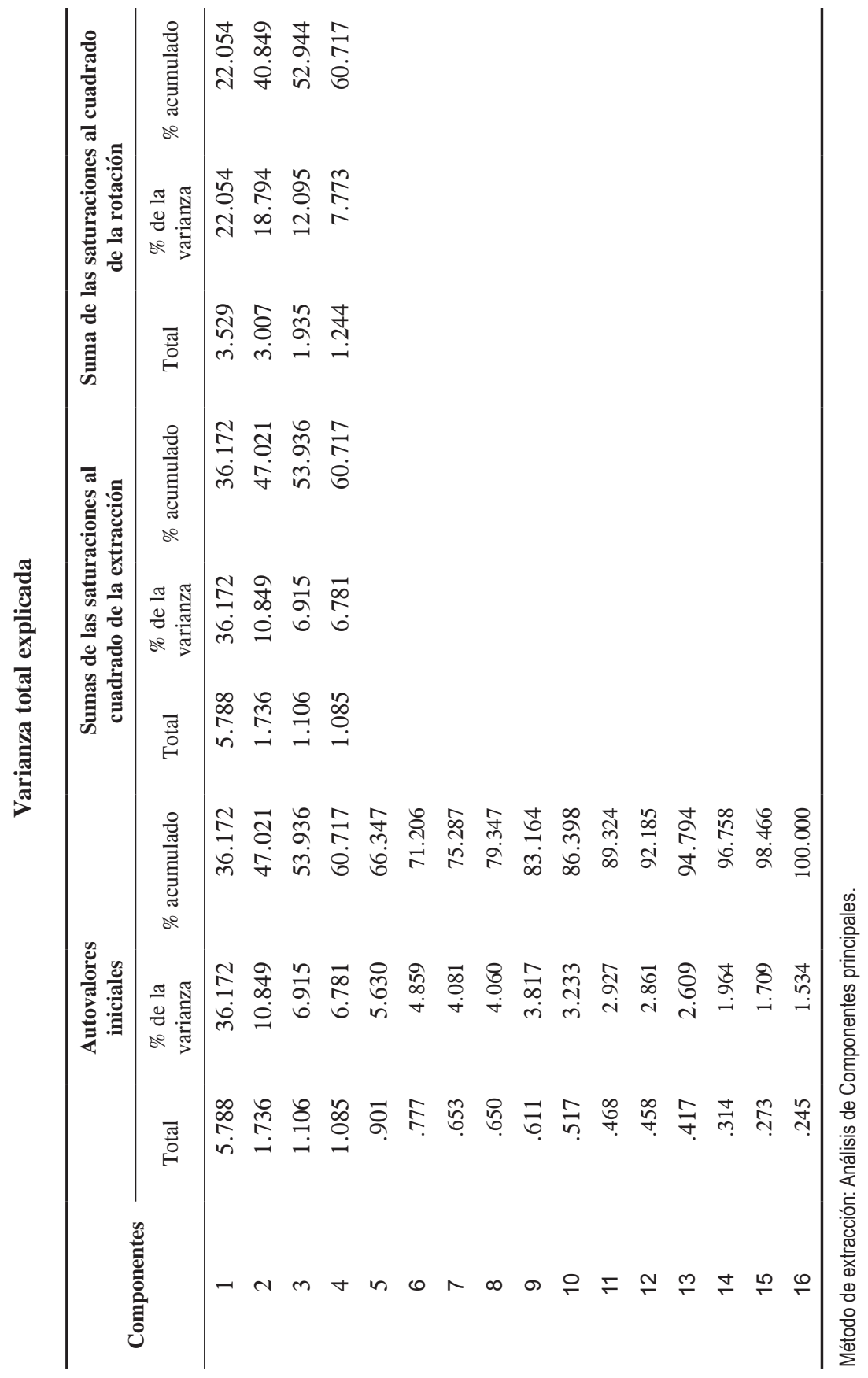


De acuerdo al análisis, la ETDPM estaría compuesta por cuatro factores siendo estos, tomados del cuadro anterior, los siguientes:

- F1: $1,2,3,4,5,11$

- F2: 8, 10, 13, 14, 15, 16

- F3: 6, 7, 9

- F4: 12

Se observa que hay un solo ítem en el cuarto factor, por ello se procede a forzar la reducción de los factores a tres.

Matriz de componentes rotados(a)

\begin{tabular}{ccccc}
\hline \multicolumn{5}{c}{ Componente } \\
\cline { 2 - 5 } & 1 & 2 & 3 & 4 \\
\hline i1 & .724 & & & \\
i2 & .785 & & & \\
i3 & .791 & & .334 & \\
i4 & .583 & & .308 & \\
i5 & .709 & & .672 & \\
i6 & & & .661 & -.313 \\
i7 & .317 & & .499 & \\
i8 & & .614 & .544 & \\
i9 & .343 & & .399 & \\
i10 & & .477 & & .778 \\
i11 & .551 & .452 & & .428 \\
i12 & & & & \\
i13 & .442 & .590 & & \\
i14 & & .723 & & \\
i15 & & .717 & & \\
i16 & & .778 & & \\
\hline
\end{tabular}

Método de extracción: Análisis de componentes principales. Método de rotación: Normalización Varimax con Kaiser.

a La rotación ha convergido en 7 iteraciones.

\section{Análisis factorial - Tres factores}

\section{Matriz de correlaciones(a)}

Determinante $=.002$

\section{KMO y prueba de Bartlett}

\begin{tabular}{llr}
\hline Medida de adecuación muestral de Kaiser-Meyer-Olkin. & .861 \\
Prueba de esfericidad de & Chi-cuadrado aproximado & 1370.314 \\
Bartlett & gl & 120 \\
& Sig. & .000 \\
\hline
\end{tabular}




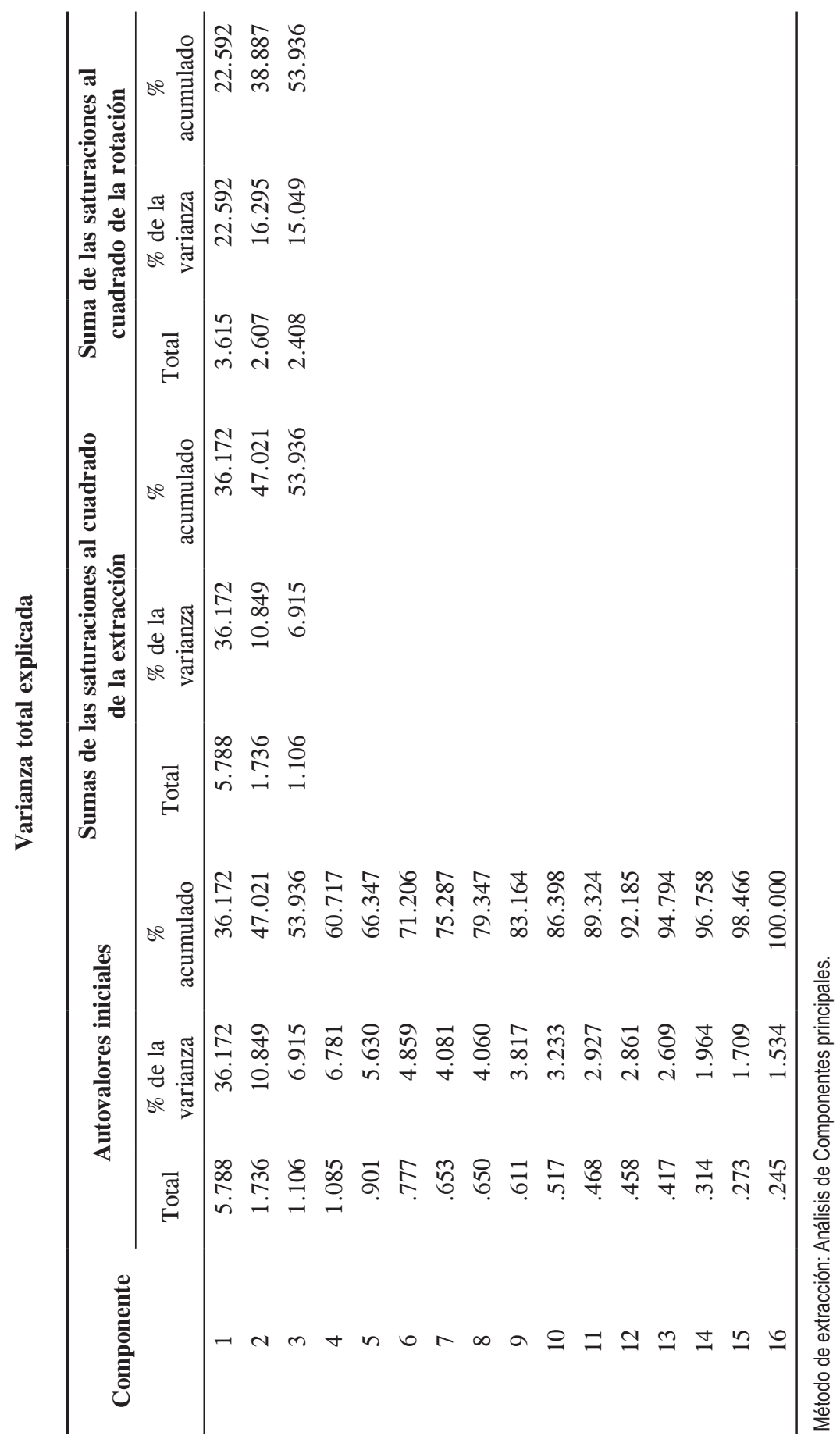


A continuación se da la nueva clasificación para tres factores:

- $\quad F 1: 1,2,3,4,5,9,11$

- $\quad F 2: 7,8,10,13,16$

- $\quad$ F3: $6,12,14,15$

Matriz de componentes rotados(a)

\begin{tabular}{|c|c|c|c|}
\hline & \multicolumn{3}{|c|}{ Componente } \\
\hline & 1 & 2 & 3 \\
\hline i1 & .748 & & \\
\hline i2 & .758 & & \\
\hline i3 & .756 & & \\
\hline i4 & .613 & & \\
\hline i5 & .755 & & \\
\hline i6 & & & .505 \\
\hline i7 & .346 & .560 & \\
\hline i8 & & .766 & \\
\hline i9 & .434 & & .414 \\
\hline i10 & & .469 & .390 \\
\hline i11 & .521 & .386 & \\
\hline i12 & & -.309 & .696 \\
\hline i13 & .377 & .518 & \\
\hline i14 & & & .745 \\
\hline i15 & & .432 & .620 \\
\hline i16 & & .674 & .410 \\
\hline
\end{tabular}

Método de extracción: Análisis de componentes principales.

Método de rotación: Normalización Varimax con Kaiser.

La rotación ha convergido en 14 iteraciones.

Estadísticos de fiabilidad de EVES

\begin{tabular}{cc}
\hline Alfa de Cronbach & $\mathbf{N}^{\circ}{ }^{0}$ de elementos \\
\hline .824 & 14 \\
\hline
\end{tabular}

Alta confiabilidad. 


\section{Estadísticos total-elemento}

\begin{tabular}{ccccc}
\hline & $\begin{array}{c}\text { Media de la } \\
\text { escala si se } \\
\text { elimina el } \\
\text { elemento }\end{array}$ & $\begin{array}{c}\text { Varianza de } \\
\text { la escala si } \\
\text { se elimina el } \\
\text { elemento }\end{array}$ & $\begin{array}{c}\text { Correlación } \\
\text { elemento-total } \\
\text { corregida }\end{array}$ & $\begin{array}{c}\text { Alfa de } \\
\text { Cronbach si } \\
\text { se elimina el } \\
\text { elemento }\end{array}$ \\
\hline a1 & 29.33 & 31.950 & .485 & .811 \\
a3 & 29.12 & 31.182 & .497 & .809 \\
a4 & 29.12 & 30.410 & .549 & .805 \\
a5 & 29.46 & 31.673 & .448 & .813 \\
a7 & 29.39 & 31.042 & .570 & .805 \\
a9 & 28.90 & 31.545 & .373 & .819 \\
a10 & 28.81 & 31.026 & .466 & .812 \\
a13 & 29.28 & 31.706 & .488 & .810 \\
a14 & 28.52 & 32.487 & .275 & .827 \\
a15 & 29.26 & 30.506 & .627 & .801 \\
a16 & 29.45 & 32.144 & .367 & .819 \\
a18 & 29.32 & 33.073 & .276 & .824 \\
a21 & 29.73 & 31.665 & .421 & .815 \\
a22 & 29.43 & 30.770 & .566 & .805 \\
\hline
\end{tabular}

Se observa que los 14 ítemes finales que componen la EVES presentan una buena correlación, por consiguiente la escala mide lo que dice medir.

\section{Análisis factorial EVES}

Matriz de correlaciones(a)

a Determinante $=.021$

KMO y prueba de Bartlett

Medida de adecuación muestral de Kaiser-Meyer-Olkin.

.833

Prueba de esfericidad de

Bartlett

Chi-cuadrado aproximado

867.336

gl

91

Sig. 


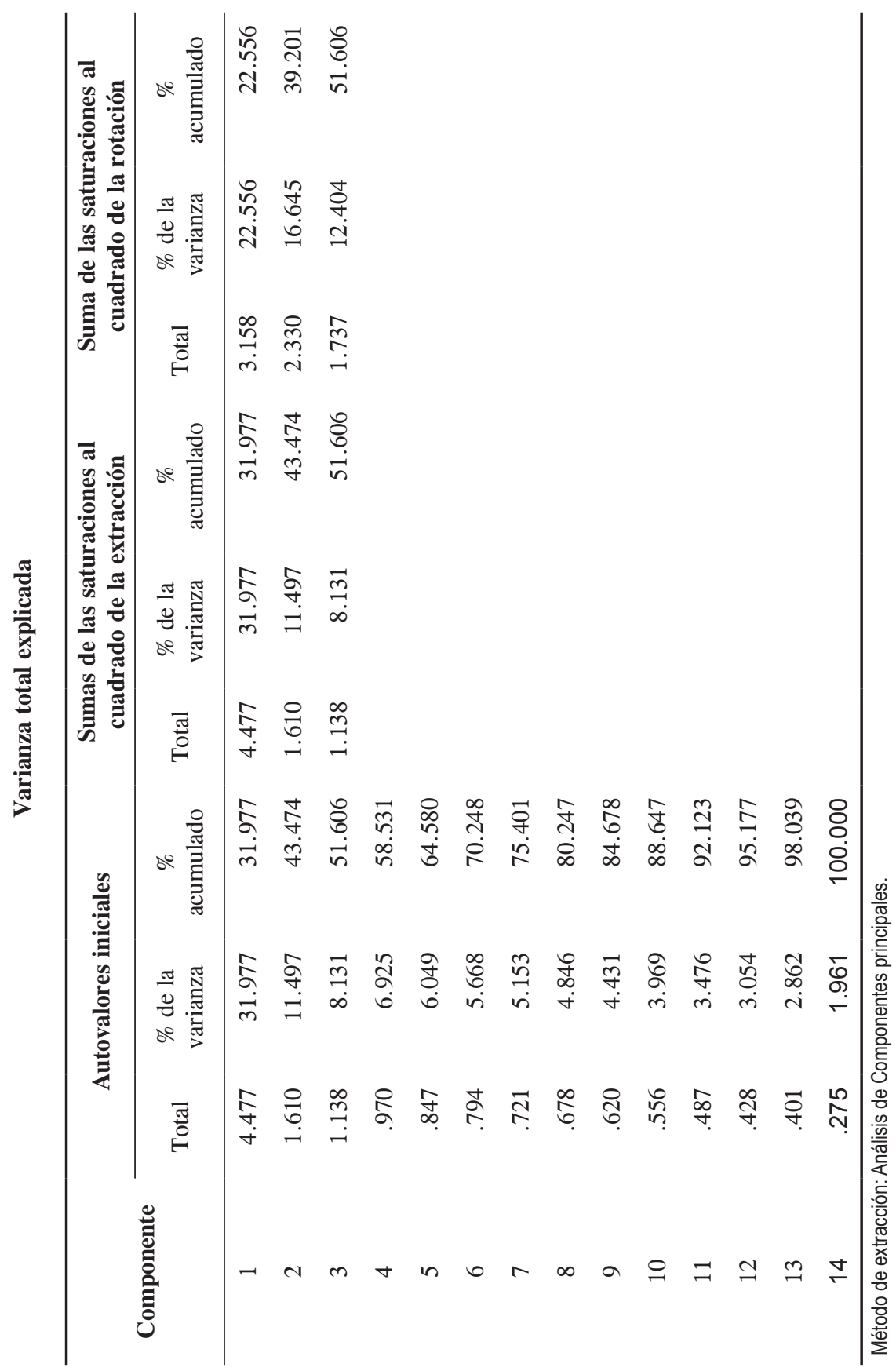


RELACIÓN ENTRE TRASTORNO DISFÓRICO PREMENSTRUAL Y ANSIEDAD, DEPRESIÓN Y ESTRÉS EN ESTUDIANTES UNIVERSITARIAS DE LA UNMSM

Matriz de componentes rotados(a)

\begin{tabular}{|c|c|c|c|}
\hline & \multicolumn{3}{|c|}{ Componente } \\
\hline & 1 & 2 & 3 \\
\hline a1 & & .706 & .328 \\
\hline $\mathrm{a} 3$ & .675 & & \\
\hline $\mathrm{a} 4$ & .715 & & \\
\hline a5 & & .809 & \\
\hline a7 & .492 & .306 & .345 \\
\hline a9 & & & .689 \\
\hline a10 & .579 & & \\
\hline a13 & & .664 & \\
\hline a14 & .547 & & -.411 \\
\hline a15 & .746 & & \\
\hline a16 & & & .611 \\
\hline a18 & & .708 & \\
\hline $\mathrm{a} 21$ & .415 & & .459 \\
\hline a22 & .641 & & \\
\hline
\end{tabular}

Método de extracción: Análisis de componentes principales. Método de rotación: Normalización Varimax con Kaiser. La rotación ha convergido en 5 iteraciones.

Los factores de esta escala son los siguientes:

F1: $3,4,7,10,14,15,22$

F2: $1,5,13,18$

F3: $9,16,21$

\section{DISCUSIÓN}

\section{Análisis de fiabilidad por factores ETDPM}

\section{Factor 1}

Estadísticos de fiabilidad factor 1

\begin{tabular}{cc}
\hline Alfa de Cronbach & $\mathbf{N}^{\mathbf{o}}$ de elementos \\
\hline .831 & 7 \\
\hline
\end{tabular}

El factor 1 de ETDPM tiene una intensidad de confiabilidad alta. 
Estadísticos de la escala

\begin{tabular}{llll}
\hline Media & Varianza & \multicolumn{1}{c}{$\begin{array}{c}\text { Desviación } \\
\text { típica }\end{array}$} & $\begin{array}{c}\text { N de } \\
\text { elementos }\end{array}$ \\
\hline 15.06 & 19.254 & 4.388 & 7 \\
\hline
\end{tabular}

\section{Análisis de fiabilidad por Factores ETDPM}

\section{Factor 2}

Estadísticos de fiabilidad Factor 2

\begin{tabular}{cc}
\hline Alfa de Cronbach & N. $^{0}$ de elementos \\
\hline .781 & 5 \\
\hline
\end{tabular}

El factor 2 de ETDPM tiene una intensidad de confiabilidad alta.

Estadísticos de la escala

\begin{tabular}{cccc}
\hline Media & Varianza & $\begin{array}{c}\text { Desviación } \\
\text { típica }\end{array}$ & $\begin{array}{c}\text { N de } \\
\text { elementos }\end{array}$ \\
\hline 11.92 & 10.090 & 3.176 & 5 \\
\hline
\end{tabular}

\section{Análisis de fiabilidad por factores ETDPM}

\section{Factor 3}

Estadísticos de fiabilidad factor 3

\begin{tabular}{cc}
\hline Alfa de Cronbach & N de elementos \\
\hline .649 & $\mathbf{4}$ \\
\hline
\end{tabular}

El factor 3 de ETDPM tiene una intensidad de confiabilidad moderada.

Estadísticos de la escala

\begin{tabular}{cccc}
\hline Media & Varianza & $\begin{array}{c}\text { Desviación } \\
\text { típica }\end{array}$ & $\begin{array}{c}\mathbf{N .}^{\mathbf{o}} \text { de } \\
\text { elementos }\end{array}$ \\
\hline 8.76 & 6.855 & 2.618 & 4 \\
\hline
\end{tabular}


La prueba en su totalidad si discrimina, cumple su cometido de medir un atributo estableciendo quiénes tienen más o menos de dicho atributo.

\section{Análisis de la fiabilidad por factores EVES}

Factor 1: síntomas ansioso-depresivos

Estadísticos de fiabilidad Factor 1: Síntomas Ansioso-Depresivos

\begin{tabular}{cc}
\hline Alfa de Cronbach & N. $^{0}$ de elementos \\
\hline .781 & 7 \\
\hline
\end{tabular}

El factor 1 de EVES tiene una intensidad de confiabilidad alta

Estadísticos de la escala

\begin{tabular}{cccc}
\hline Media & Varianza & $\begin{array}{c}\text { Desviación } \\
\text { típica }\end{array}$ & $\begin{array}{c}\mathbf{N .}^{\mathbf{0}} \text { de } \\
\text { elementos }\end{array}$ \\
\hline 16.65 & 13.164 & 3.628 & 7 \\
\hline
\end{tabular}

Análisis de fiabilidad por Factores EVES

\section{Factor 2: Déficit cognitivo}

Estadísticos de fiabilidad Factor 2: Déficit cognitivo

\begin{tabular}{cc}
\hline Alfa de Cronbach & N. $^{\circ}$ de elementos \\
\hline .733 & 4 \\
\hline
\end{tabular}

El factor 2 de EVES tiene una intensidad de confiabilidad alta.

Estadísticos de la escala

\begin{tabular}{cccc}
\hline Media & Varianza & $\begin{array}{c}\text { Desviación } \\
\text { típica }\end{array}$ & $\begin{array}{c}\mathbf{N .}^{\mathbf{0}} \text { de } \\
\text { elementos }\end{array}$ \\
\hline 8.50 & 4.635 & 2.153 & 4 \\
\hline
\end{tabular}




\section{Análisis de fiabilidad por Factores EVES}

Factor 3: Síntomas físicos

Estadísticos de fiabilidad factor3

\begin{tabular}{cc}
\hline Alfa de Cronbach & N. $^{\mathbf{0}}$ de elementos \\
\hline .534 & $\mathbf{3}$ \\
\hline
\end{tabular}

El factor 3 de EVES tiene una intensidad de confiabilidad moderada.

Estadísticos de la escala

\begin{tabular}{cccc}
\hline Media & Varianza & $\begin{array}{c}\text { Desviación } \\
\text { típica }\end{array}$ & $\begin{array}{c}\mathbf{N .}^{\mathbf{0}} \text { de } \\
\text { elementos }\end{array}$ \\
\hline 6.33 & 3.173 & 1.781 & 3 \\
\hline
\end{tabular}

\section{Correlaciones}

\section{Correlaciones}

\begin{tabular}{llrr}
\hline & & Total_ETPDM & Total_newEVES \\
\hline Total_ETPDM & Correlación de Pearson & 1 & $.653(* *)$ \\
& Sig. (bilateral) & & .000 \\
& $\mathrm{~N}$ & 230 & 230 \\
Total_newEVES & Correlación de Pearson & $.653(* *)$ & 1 \\
& Sig. (bilateral) & .000 & \\
& $\mathrm{~N}$ & 230 & 230 \\
\hline
\end{tabular}

** La correlación es significativa al nivel 0,01 (bilateral).

Sí hay correlación entre ETPDM y EVES y es significativa.

\section{CONCLUSIONES}

- La escala EVES fue adaptada y validada exitosamente a nuestro medio y está constituida por ítemes agrupados en tres factores:

- Síntomas Ansioso-Depresivos: Ítemes 3,4,7,10,14,15,22

- Déficit cognitivo: Ítemes $1,5,13,18$

- Síntomas físicos: Ítemes 9,16,21

Su alfa de Cronbach es de 0.824 . 
- La escala ETDPM está constituida por 16 ítemes los cuales se agrupan en tres factores:

- Desequilibrio Emocional : Ítems 1,2,3,4,5,9,11 (F1)

- Cansancio Físico.: Items 7,8,10,13,16 (F2)

- Somatización.: Items 6,12,14,15 (F3)

Siendo su alfa de Cronbach de 0.877 .

- $\quad$ La correlación de ambas escalas presenta una correlación de Pearson de 0.653.

- Las mujeres evaluadas presentan un predominio de dolores premenstruales a partir de 19-23 años de edad. Se debe informar las acciones psicológicas para que dicho ciclo no se vea alterado con manifestaciones de un desajuste emocional, de esta manera estaremos realizando prevención primaria.

- Se confirman los datos estadísticos a nivel internacional que la menstruación tiene inicio a partir de los 11 años de edad cronológica.

- Se hace notorio en la presente investigación que, a partir de los 11 hasta los 14 años de edad, es necesario trabajar mancomunadamente con los ginecólogos, biólogos, psiquiatras, antropólogos y psicólogos para determinar la incidencia y prevalencias de los dolores menstruales.

- En más del 50\% de la muestra, la duración de su ciclo menstrual es de 3 a 4 días, también se hace evidente que aproximadamente un $33 \%$ no presenta un ciclo menstrual regular.

- Los datos estadísticos de la presente investigación nos indican que los dolores menstruales se presentan en las mujeres cuyos periodos tienen una duración de 3 - 5 días o más, siendo necesario trabajar con un equipo interdisciplinario para determinar las causas psicológicas, biológicas y sociales que actúan en la persistencia de dichos dolores.

- Se necesita hacer investigaciones interdisciplinarias para ampliar el presente estudio.

\section{REFERENCIAS BIBLIOGRÁFICAS}

1. Bacstrom (1992). Neuroendocrinología del síndrome premenstrual. Clínicas obstétricas y ginecológicas.

2. Bernard, J. (1977). Celos y matrimonio. Englewood Cliffs, New Jersey: Prentice Hall.

3. De Gregorio, S. (1996). La depresión en las mujeres. Un enfoque desde la biología de los trastornos del estado de ánimo. Revista Argentina de Psiquiatría Biológica.

4. Ibañez (1996). Endocrinopsiquiatría del síndrome premenstrual. Revista Argentina de Psiquiatría biológica. 
5. Lolas J. (1995). Síndrome premenstrual desde una nueva perspectiva. Chile: Edit. Texido.

6. Mortola J. (1992). Aspectos diagnósticos y de investigación de síndrome premenstrual. Clínica Obstétrica y ginecológica.

7. Peralstein T., Stone (1998). A Premenstrual Syndrome. Psychiatric Clinics North América.

8. Resding A. (1992). Modelo cognoscitivo del síndrome premenstrual. Clínica obstétrica y ginecológica.

9. Telles J., Gaviria S., Burgos C. (1999). El síndrome premenstrual. En: Afrodita y Esculapio. Santa Fe de Bogotá: Ed. Nuevo Milenio. 